\title{
Observed characteristics of luminous variation events during the initial stage of upward positive leaders
}

\author{
D.Wang, N.Takagi, T.Watanabe \\ Gifu University, Yanagito 1-1, Gifu, Japan
}

\begin{abstract}
We have measured the correlated sub-microsecond E-fields and high-speed images of the luminous variation events during the initial stage of three upward positive leaders initiated from a high tower. We found that each of the luminous variation events is composed of many electrical breakdowns. We classified the luminous variation events into the following two types: (1) they appear with an initial dominant pulse and their propagations can be identified from high speed images; (2) there is not an initial dominant pulse and their propagations can not be clearly identified. We inferred that in terms of propagation the luminous variation events are similar to ICC (initial continuous current) pulses and M-components. Based on the measured results and as an extension, we have discussed the contradicted propagation characteristics of M-components, an issue which has remained confusing in literatures for many years. We speculated that (1) an M-component is a composition of many waves occurring at different times and different locations, and those waves may interfere each other complicatedly; (2) an M-component may involve some waves excited locally anywhere along its continuous current channel through concentrating or absorbing electrical energy from ambient electric field; (3) the speed of an M-component, if measured optically by assuming that it is a process of only one or two waves, may be , in most of cases, just a kind of apparent and artificial (not real in physics) speed produced by many factors such as instrument sensitivity, and at which channel section and how the speed is measured. All these aspects together account for the contradicted propagation characteristics of M-components.
\end{abstract}

Key words: lightning discharge, leader, M-component

\section{Introduction}

Upward positive leaders have been observed with high speed images by many authors (e.g., Berger, 1967; Idone 1992). An apparent feature of upward positive leaders is its channel luminous intensity variation phenomena. Such luminosity variations are likely to be simply interpreted as leader stepping, but for most of the luminosity variations, they are remarkably different from leader stepping. Leader stepping, especially the stepping of negative leaders, usually shows a sharp image over a small portion of channel (e.g., Wang et al., 1999b), while most of the luminosity variation events exhibit diffuse images over whole leader channels. The detailed characteristics of such luminous variations are unknown. For this reason, we have measured the correlated sub-microsecond E-fields and high-speed images of the luminous variation phenomena during the initial stage of upward positive leaders from a high tower. The first objective of this short paper is to present our observed characteristics of the luminous variation events (LVE).

On the other hand, from the observed characteristics, we inferred that the luminous variation events are very similar to $M$-components which are the temporary increase phenomena in luminosity of the lightning luminous channel or in relatively steady continuing lightning current following return strokes (Malan and Collens, 1937). As pointed out by 
Rakov et al. (1995), although M-component has been studied by a number of authors (e.g., Malan and Schonland, 1947; Kitagawa et al., 1962; Thottappillil et al., 1990; 1995; Rakov et al., 1992;1995; Jordan et al., 1995) during the past several tens of years, compared with leaders and return strokes, the propagation characteristics of M-components measured through high speed imaging systems were very confusing, with some M-components being reported to propagate downward, some upward and some with indeterminate directions. Based on the analysis of simultaneous measurements of the channel-base current and the vertical electric field $30 \mathrm{~m}$ from rocket-triggered lightning, Rakov et al. (1995) proposed a two-wave theory to explain such contradiction. Our measured results appear to provide additional hints to understand the contradicted propagation characteristics of M-components. As an extension, the second objective of this paper is to present a short discussion on the propagation of M-components.

\section{Observation and the Results}

Our observation was performed with a new version of ALPS (Automatic Lightning Progressing Feature Observation System) that has been described in detail by Wang et al. (2004). For the present study, the lightning E-field change, output from two capacitive E-field antennas which have time constants of $2.2 \mathrm{~s}$ and $1.5 \mathrm{~ms}$, respectively, was simultaneously recorded with the ALPS system at a time of resolution $100 \mathrm{~ns}$. The antenna with a time constant of $2.2 \mathrm{~s}$ is called as slow antenna and the one with a time constant of $1.5 \mathrm{~ms}$ is called as fast antenna. The slow antenna covers a frequency bandwidth from a few $\mathrm{Hz}$ to around 1 $\mathrm{MHz}$ and the fast antenna covers a bandwidth from several hundred $\mathrm{Hz}$ to around $5 \mathrm{MHz}$. We chose the tip of a $60 \mathrm{~m}$ high tower, which was built at the top of a mountain $270 \mathrm{~m}$ high above the sea level, as the view target of ALPS. The mountain locates in Hokuriku area of Japan, a place famous for winter lightning. The ALPS was installed in a building about $1 \mathrm{~km}$ away from the tower, and the resultant ALPS spatial resolution is around $25 \mathrm{~m}$. The two E-field change antennas were set up at the roof of the building. The time resolution of ALPS system was set at $100 \mathrm{~ns}$ and the resultant recording time is $3.2 \mathrm{~ms}$.

The correlated E-fields and high-speed images of three leaders initiated naturally from a tower were recorded on 13 December 2001. Since all three leaders exhibit similar characteristics, for simplicity, we just present the observed waveforms for the leader occurring at 22:53:15 (local time) as seen in Fig. 1 and Fig. 2. Figure 1 shows the relative light intensity waveforms as a function of time at various heights from S1 (tower tip) through S12 (260 m) as well as the correlated E fields. All the signals are in relative units. As evident in Fig. 1, the leader light signals at the lower heights occur earlier in time, hence this leader propagates in upward direction. The average speed estimated over the entire $260 \mathrm{~m}$ channel bottom for the leader is $6.5 \times 10^{5} \mathrm{~m} / \mathrm{s}$. From the polarity of E-field change as shown in Fig. 1, the leader is identified to be positive. Also seen in Fig. 1, at least 8 channel luminosity variation events can be identified in the light signals of the leader and they are named as $\mathrm{LVE}_{1}, \mathrm{LVE}_{2}, \mathrm{LVE}_{3}, \mathrm{LVE}_{4}$, $L_{V E}, L V E_{6}, L V E_{7}$ and $L V E_{8}$, respectively. Those luminosity variation events can be roughly classified into two types from their rise times and propagation features. Figure $2 \mathrm{~A}$ shows an example of the first type event (LVE 2 in Fig. 1). Small regular pulses seen in the signals are due to instrument noises. $\mathrm{LVE}_{2}$ can be clearly identified at the heights from S1 (tower tip) to $\mathrm{S} 5(90 \mathrm{~m})$ with a rise time of about $30 \mu \mathrm{s}$. Judging from the onset times of $\mathrm{LVE}_{2}$ at different heights, neither propagation direction nor speed can be determined for this type of events. During the whole stage of $\mathrm{LVE}_{2}$, as evident in Fig.2A, many pulses can be identified in its 


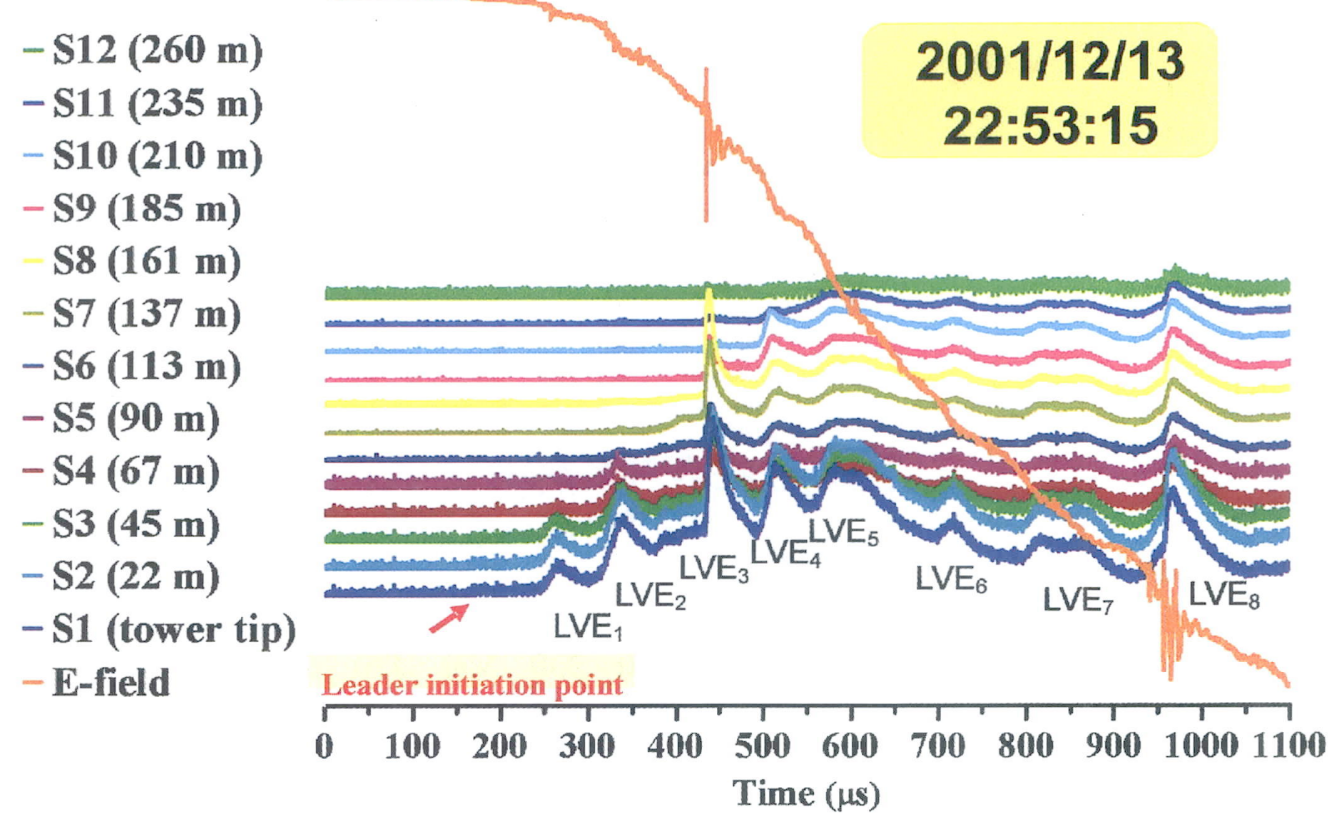

Fig. 1 Light intensity waveforms as a function of time at various heights from S1 (tower tip) through S12 $(260 \mathrm{~m})$ and the correlated fields for an upward leader initiated from a high tower at 2253:42 on December 13, 2001 at Hokuriku area of Japan. All the waveforms are in relative units. Eight luminous variation events (LVE) from $\mathrm{LVE}_{1}$ to $\mathrm{LVE}_{8}$ can be seen in the light waveforms.

corresponding E-field signal. These pulses may imply that $\mathrm{LVE}_{2}$ is actually a composite effect of many electrical breakdowns which occur at different time and by inference at different locations. Figure 2B shows an example of the second type luminosity variation events $\left(\mathrm{LEV}_{3}\right.$ in Fig. 1). As seen in Fig. 2B, $\mathrm{LVE}_{3}$ appears with an initial dominant light pulse having a peak at S8. Judging from the onset time of the dominant pulse, $\mathrm{LVE}_{3}$ starts between S8 and S9 and then propagates toward S6 at a speed close to $1 \times 10^{8} \mathrm{~m} / \mathrm{s}$. From $\mathrm{S} 5$, the dominant pulse becomes indistinguishable and the propagation direction of $\mathrm{LVE}_{3}$ becomes indeterminate. The rise time of the dominant pulse measured at $\mathrm{S} 8$, between the two arrows as shown in Fig. $2 \mathrm{~B}$, is $0.6 \mu$ s. Followed this initial dominant pulse, similar to $\mathrm{LVE}_{2}$, a few pulses can be identified in the corresponding E-field signal of $\mathrm{LVE}_{3}$. Again, these pulses imply that $\mathrm{LVE}_{3}$ is also a composition of many electrical breakdowns although its first breakdown is a dominant one as seen in Fig. 2B.

Totally, 22 pronounced LEV are identified in the initial stages (within $1 \mathrm{~ms}$ from the leader initiation) of the three leaders observed in this study. Among the $22 \mathrm{LEV}$, three are classified as type 1 and the remaining 19 as type 2 . A statistical study has been performed for LVE 10-90 \% risetime, duration and intervals and the results are shown in Fig. 3. As seen in this figure, the rise time ranges from $0.6 \mu$ s to $77.6 \mu$ s with a geometric mean (GM) value of $11.9 \mu \mathrm{s}$; the time duration distributes from $7.8 \mu \mathrm{s}$ to $173.7 \mu \mathrm{s}$ with a GM value of $76.9 \mu \mathrm{s}$; the 


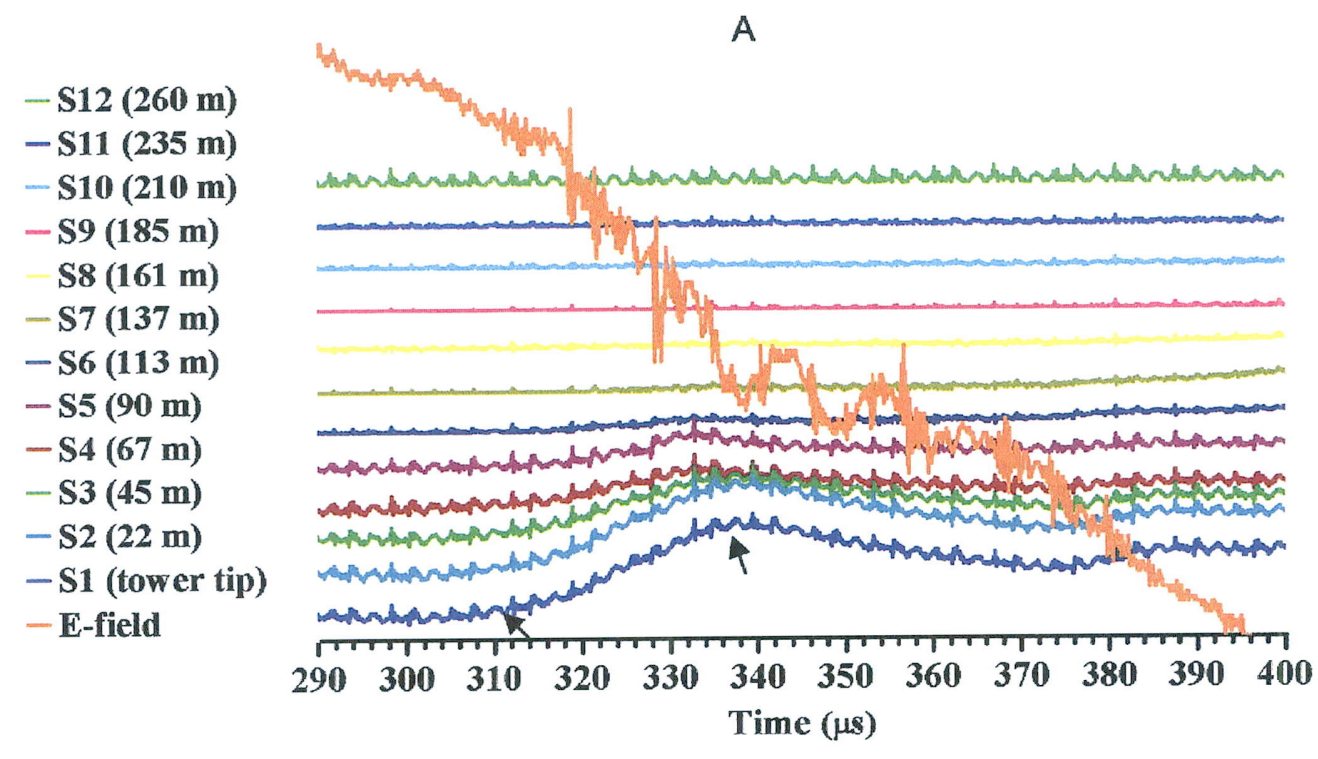

B

$-\mathrm{S} 12(260 \mathrm{~m})$
$-\mathrm{S} 11(235 \mathrm{~m})$
$-\mathrm{S} 10(210 \mathrm{~m})$
$-\mathrm{S} 9(185 \mathrm{~m})$
$-\mathrm{S} 8(161 \mathrm{~m})$
$-\mathrm{S} 7(137 \mathrm{~m})$
$-\mathrm{S} 6(113 \mathrm{~m})$
$-\mathrm{S} 5(90 \mathrm{~m})$
$-\mathrm{S} 4(67 \mathrm{~m})$
$-\mathrm{S} 3(45 \mathrm{~m})$
$-\mathrm{S} 2(22 \mathrm{~m})$
$-\mathrm{S} 1($ tow er tip)
$-\mathrm{E}-$ field
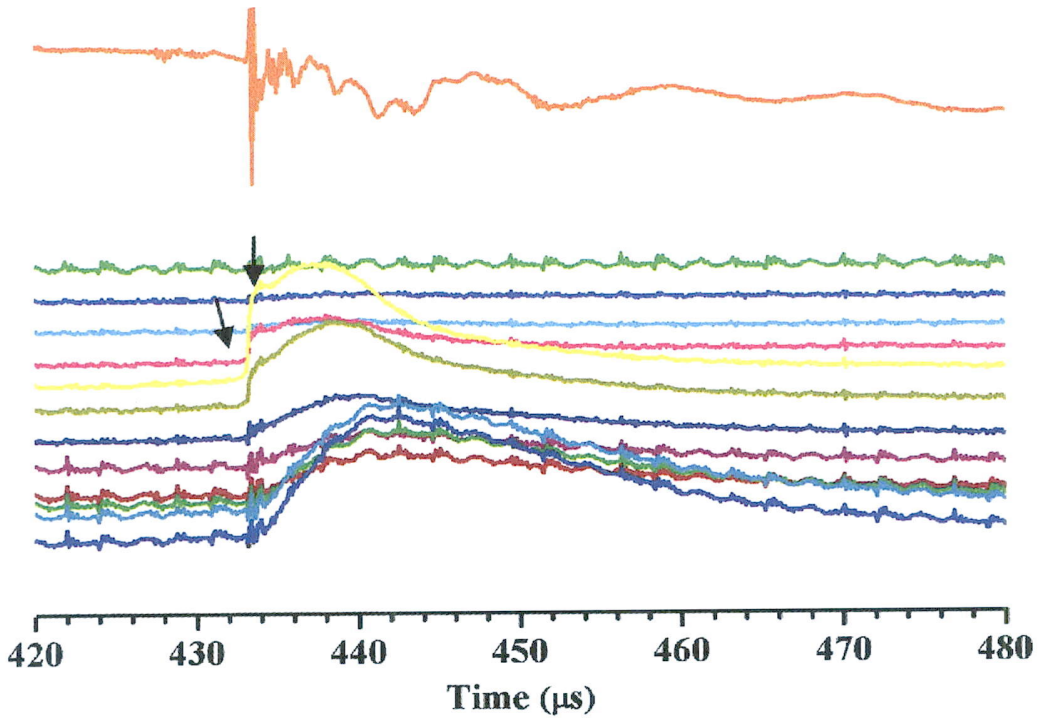

Fig. 2 Expanded waveforms of Fig 1. (A) is for $\mathrm{LVE}_{2}$ and (B) is for $\mathrm{LVE}_{3}$. $\mathrm{LVE}_{3}$ contains an initial dominant pulse and $\mathrm{LVE}_{2}$ does not. Small regular pulses are instrument noises.

time interval varies from $67.8 \mu$ s to $190.3 \mu$ s with a GM value of $97.7 \mu$ s. Since the optical pulse of a leader step usually has a rise time less than $1 \mu$ s (Wang et al., 1999b), apparently, in 
most of the cases, LVE has a rise time much larger than that of leader steps.

\section{Discussion}

(a) Time-resolved photographic records of the luminous variation events during the initial stage of positive upward leaders have been presented by Idone (1992), Wada et al. (1996) and Rakov et al. (2003). All these authors suggested that the LVE are associated with leader steps. Idone (1992) reported that particularly well developed illuminations propagate back down the channel in association with the individual step pulses. By using two of the most pronounced illuminations, Idone (1992) estimated the lower bound of the propagation speeds being $5 \times 10^{7} \mathrm{~m} / \mathrm{s}$ and $6 \times 10^{7} \mathrm{~m} / \mathrm{s}$. LVE propagation can not be identified from the high-speed records presented by Wada et al. (1996) and Rakov et al. (2003). Our data indicate that if a LVE contains an initial dominant pulse, its propagation speed can be obtained, otherwise even its propagation direction can not be determined. Meanwhile, as reported above, LVE has a rise time much larger than that of leader steps. All these facts suggest that LVE can not be simply classified into leader steps.

(b) A simple comparison between the LVE and M-components has been made and several significant similarities between them can be noted as follows: (a)

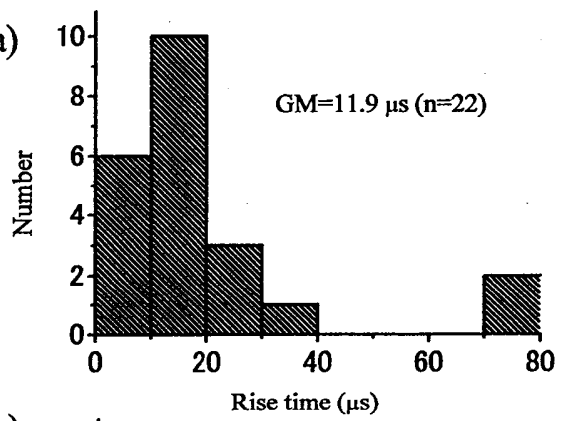

(b)

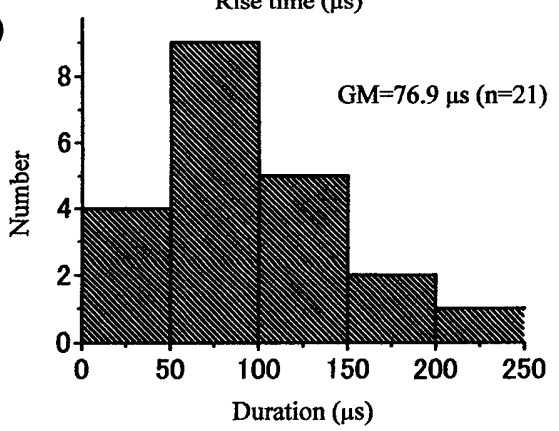

(c)

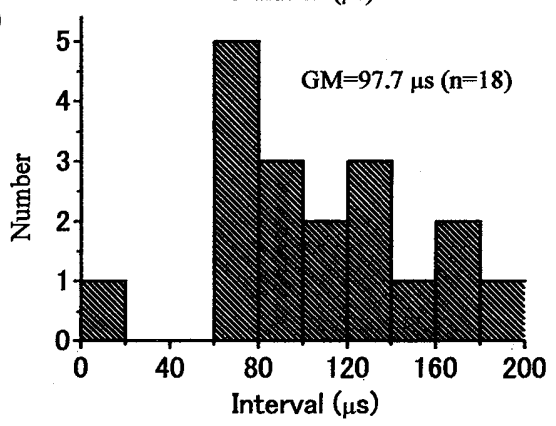

Fig. 3 Histograms of the LVE 10-90\% risetime (a), duration (b) and time interval (c).

(1) both of them are temporary channel-luminosity-increase phenomena; (2) both of them usually have a slower rise time than a stepped-leader pulse and a return stroke; (3) in most of the cases even their propagation directions are indeterminate. These similarities lead us to have a short discussion on the contradicted propagation characteristics of M-components which remained unsolved for many years. Based on our observed results on LVE, we speculate that, depending on whether there is a dominant pulse as onset, M-components can be classified into the following three cases. (i ) When an M-component is the manifestation for a conductive lightning channel to approach a space charge region, numerous breakdowns that occur at different times could be involved. All these breakdowns propagate as current waves along the lightning channel and may interfere each other in complicated ways. When the waves encounter any dissimilarity in conductivity along the channel, reflected waves should occur 
(e.g., Rakov et al., 1995). Further and more importantly, since the ambient electric field is not zero during an M-component and the electromagnetic radiation waves (energy) by the M-component itself is propagating in space with a speed of light, any channel section with conductivity could locally absorb electrical energy from the ambient electric field and the radiation waves through electrical induction. It is not difficult to speculate that such absorbed energy will accumulate somewhere and eventually excite some types of waves. The well-known phenomenon of upward connecting leaders which are initiated from a lightning rod or any other tall conductive structures should be a kind of related and likely the most apparent evidence for such local waves. When a downward leader is approaching closely to a lightning rod, the lightning rod, through electrical induction, adsorbs and concentrates the low frequency electromagnetic waves radiated from the coming downward leaders, and then excites locally on the tip of lightning rod a breakdown wave propagating upwardly (an upward leader). Although the waves excited locally along an M-component channel could appear differently in comparison with the upward leaders, an M-component is a composite effect of all the waves occurred along its channel. Since the waves are produced at different times and, by inference, at different locations, and may have different characteristics, and even suffer nonlinear interference, the speed of an M-component, if measured optically by assuming that it is a process of only one or two waves, may be just a kind of apparent and artificial (not real in physics) speed produced by many factors such as instrument sensitivity, and at which channel section and how the speed is measured. (ii) When an M-component occurs as a contact of an in-cloud conducting channel with a conducting channel to ground, a dominant breakdown occurs first and then is followed by small breakdowns for transferring nearby space charges. Similarly, many waves could be produced and an M-component is a composition of all these waves occurring at different times and different locations. In this case, the M-component will appear to propagate downward at its initial stage due to the dominant breakdown pulse. After the dominant pulse becomes indistinguishable, the propagation characteristics become similar to that in case ( i ). Rakov et al. (1992) and Shao et al. (1995) reported some electric field pulses at the initial stage of M-components. These kinds of fast electric field variations may correspond to the initial dominant breakdowns, which are similar to that shown in Fig.2B. (iii) if the first dominant pulse discharge dominates until to ground, then a discharge analogous to a return stroke may be initiated. In this case, first a downward and then an upward propagation may be observed as shown by Rakov and Uman (2003, P.271, Fig.7.5). Based on high-speed photographic measurements, Malan and Collens (1937) reported that seven M-components recorded by them definitely travel downward while two appear to travel upward. We speculate that the downward M-components reported by them correspond to case (ii) and the upward ones to case (iii).

Through high-speed optical measurements, Wang et al. (1999c) reported some complicated phase velocities of $\mathrm{M}$-components in rocket triggered lightning. It appears that their results agree with our speculations. Also through high-speed optical measurements, Wang et al. (1995) reported some bidirectional discharges near the top of the $\mathrm{CN}$ tower. Based on the above speculations, we infer that most of the bidirectional discharges may be just a kind of initial continuous pulses (ICC pulses) which are reported to have similar characteristics of $\mathrm{M}$-components and what they measured are kind of apparent and artificial propagations.

To explain the contradicted propagation characteristics of M-components, Rakov et al. $(1995,2001)$ proposed that a M-component involves a downward progressing incident wave (the analog of a leader) followed by an upward progressing reflected waive (the analog of a 
return stroke). Apparently, if our speculation as presented above is correct, the two-wave theory would be too simple and more physical processes should be included in future work.

(c) The composite effect of many waves as described above for M-components may be a common feature in lightning discharges. As a matter of fact, Wang et al. (2006) have pointed out that similar effects exist in return strokes. Similar effects should exist in other well documented phenomena, such as K-changes (Kitagawa, 1957). Regarding to these issues, further study is needed.

\section{Conclusion}

We have measured the correlated sub-microsecond E-fields and high-speed images of the luminous variation events during the initial stage of three upward positive leaders initiated from a high tower. We found that each of the luminous variation events is composed of many electrical breakdowns. We classified the luminous variation events into the following two types: (1) they appear with an initial dominant pulse and their propagations can be identified from high speed images; (2) there is not an initial dominant pulse and their propagations can not be clearly identified. We inferred that the luminous variation events are similar to ICC (initial continuous current) pulses and M-components. Based on the measured results and as an extension, we have discussed the contradicted propagation characteristics of $\mathrm{M}$-components. We speculated that (1) an M-component is a composition of many waves occurring at different times and different locations, and those waves may interfere each other complicatedly; (2) an M-component may involve some waves excited locally anywhere along its continuous current channel through concentrating or absorbing electrical energy from ambient electric field; (3) the speed of an M-component, if measured optically by assuming that it is a process of only one or two waves, may be , in most of cases, just a kind of apparent and artificial (not real in physics) speed produced by many factors such as instrument sensitivity, and at which channel section and how the speed is measured.

\section{Acknowledgement}

This work was supported by Grand-in-Aid for Scientific Research of the Ministry of Education, Science, Sports and Culture, Japan.

\section{References}

Berger, K. (1967), Novel observations on lightning discharges. J.Franklin Inst., 283:478-525.

Idone, V.P. (1992), The luminous development of Florida triggered lightning, Res.Lett. Atmos. Electr. 12, 23-28.

Jordan, D. M., V. P. Idone, R. E. Orville, V. A. Rakov, and M. A. Uman (1995), Luminosity characteristics of lightning M components, J. Geophys. Res. 100, 25695-256700.

Kitagawa, N. (1957), On the mechanism of cloud flash and junction or final process in flash to ground, -study of lightning discharge (II), Meteorology and Geophysics, Vol. V II, No.4,415-424.

Kitagawa, N., M. Brook, and E. J. Workman (1962), Continuing currents in cloud-to-ground lightning discharges, J. Geophys. Res. 67, 637-647.

Malan, D.J., and H. Collens (1937), Progressive lightning III - the fine structure of return lightning strokes, Proc. Roy. Soc. A 162, 175-203.

Malan, D.J., and B.F.J. Schonland (1947), Progressive lightning, Part 7, Directly correlated photographic and electrical studies of lightning from near thunderstorms, Proc. Roy. Soc. A 191, 
485-503.

Rakov, V. A., R. Thottappillil, and M. A. Uman (1992), Electric field pulses in K and M changes of lightning ground flashes, J. Geophys. Res. 97, 9935-9950.

Rakov, V. A., R. Thottappillil, M. A. Uman, and P. P. Barker (1995), Mechanism of the lightning M-component, J. Geophys. Res. 100, 25701-25710.

Rakov, V. A., D.E. Crawford, K.J. Rambo, G. H. Schnetzer, M. A. Uman, and R. Thottappillil (2001), M-component mode of charge transfer to ground in lightning discharges, J. Geophys. Res. 106, 22817-22831.

Rakov, V. A., D.E. Crawford, V. Kodali, V. P. Idone, M. A. Uman, G. H. Schnetzer, and K.J. Rambo, (2003), Cutoff and reestablishment of current in rocket-triggered lightning, J. Geophys. Res. 108, No.D23, 4747, doi:10.1029/2003JD003694.

Rakov, V.A., and M.A. Uman (2003), Lightning Physics and Effects, Cambridge University Press, pp.176-182.

Shao, X. M. , P. R. Krehbiel, R. J. Thomas, and W. Rison (1995), Radio interferometric observation of cloud-to-ground lightning phenomena in Florida, J. Geophys. Res., 100, 2749-2483.

Thottappillil, R. V. A. Rakov, and M. A. Uman (1990), K and M changes in close lightning ground flashes in Florida, J. Geophys. Res. 95, 18631-18640.

Thottappillil, R., J.D. Goldberg, V. A. Rakov, and M. A. Uman (1995), Properties of M components from currents measured at triggered lightning channel base, J. Geophys. Res. 100, 25711-25720.

Wada, A., A. Asakawa, and T. Shido (1996), Characteristics of lightning flash initiated by an upward leader in winter, Paper presented at 23rd International Conference on Lightning Protection, Italy.

Wang, D., Z-I. Kawasaki, K. Yamamoto, K. Matsuura, J. Chang, and W. Janischewskyj (1995), Luminous propagation of lightning attachment to CN tower, J. Geophys. Res. 100, 11661-11667.

Wang, D., V.A.Rakov, M.A.Uman, M.I.Fernandez, K.J.Rambo, G.H.Schnetzer, R.J.Fisher (1999a), Characterization of the initial stage of negative rocket-triggered lightning, J. Geophys. Res., Vol.104, pp.4213-4222.

Wang, D., N.Takagi, T.Watanabe, V.A. Rakov, and M.A. Uman (1999b), Observed leader and return-stroke propagation characteristics in the bottom $400 \mathrm{~m}$ of a rocket-triggered lightning channel, J.Geophys.Res., 104, 14369-14376.

Wang, D., T.Ito, N. Takagi, T. Watanabe, V. Rakov, M. Uman (1999c), Propagation characteristics of return stroke and $\mathrm{M}$-components in Florida rocket-triggered lightning, Proceedings of 11th International Conference on Atmospheric Electricity, pp. 99-102.

Wang, D., N. Takagi, X. Liu, T. Watanabe, and A. Chihara (2004), Luminosity characteristics of multiple dart leader/return stroke sequences measured with a high-speed digital image system, Geophys. Res. Lett., Vol.31, L02111, doi:10.1023/2003GL018613.

Wang, D., V. A. Rakov, N. Takagi, and T. Watanabe (2006), Common features of return stroke optical traveling waves and their interpretations, Proceedings of 28th International Conference on Lightning Protection, 257-260.

(Received October 24, 2006; revised January 20, 2007; accepted January 31, 2007) 